\title{
Bistability, Causality, and Complexity in Cortical Networks: An In Vitro Perturbational Study
}

\author{
Mattia D'Andola ${ }^{1}$, Beatriz Rebollo ${ }^{1}$, Adenauer G. Casali ${ }^{2}$, Julia F. Weinert ${ }^{1}$, \\ Andrea Pigorini ${ }^{3}$, Rosa Villa ${ }^{4,5}$, Marcello Massimini ${ }^{3,6}$ \\ and Maria V. Sanchez-Vives ${ }^{1,7}$
}

${ }^{1}$ IDIBAPS (Institut D’Investigacions Biomèdiques August Pi i Sunyer), Roselló 149-153, 08036 Barcelona, Spain, ${ }^{2}$ Federal University of São Paulo, Institute of Science and Technology, Av. Cesare Monsueto Giulio Lattes, 1211 - Jardim Santa Ines I, São José dos Campos - SP 12231-280, Brazil, ${ }^{3}$ Department of Biomedical and Clinical Sciences "L. Sacco", via G. B. Grassi 74 - Università degli studi di Milano, 20157 Milano, Italy, ${ }^{4}$ Instituto de Microelectrónica de Barcelona (IMB-CNM), CSIC, Campus UAB, 08913 Bellaterra, Barcelona, Spain, ${ }^{5}$ CIBER de Bioingeniería, Biomateriales y Nanomedicina (CIBER-BBN), 08913 Barcelona, Spain, ${ }^{6}$ Istituto Di Ricovero e Cura a Carattere Scientifico, Fondazione Don Carlo Gnocchi, 20148 Milan, Italy and ${ }^{7}$ ICREA, ICREA Passeig Lluís Companys 23, 08010 Barcelona, Spain

Address correspondence to Maria V. Sanchez-Vives, Email: msanche3@clinic.ub.es

Marcello Massimini and Maria V. Sanchez-Vives are considered as a Co-senior authors

\begin{abstract}
Measuring the spatiotemporal complexity of cortical responses to direct perturbations provides a reliable index of the brain's capacity for consciousness in humans under both physiological and pathological conditions. Upon loss of consciousness, the complex pattern of causal interactions observed during wakefulness collapses into a stereotypical slow wave, suggesting that cortical bistability may play a role. Bistability is mainly expressed in the form of slow oscillations, a default pattern of activity that emerges from cortical networks in conditions of functional or anatomical disconnection. Here, we employ an in vitro model to understand the relationship between bistability and complexity in cortical circuits. We adapted the perturbational complexity index applied in humans to electrically stimulated cortical slices under different neuromodulatory conditions. At this microscale level, we demonstrate that perturbational complexity can be effectively modulated by pharmacological reduction of bistability and, albeit to a lesser extent, by enhancement of excitability, providing mechanistic insights into the macroscale measurements performed in humans.
\end{abstract}

Key words: bistability, cerebral cortex, complexity, consciousness, slow oscillations

\section{Introduction}

An emerging idea in theoretical neuroscience is that complex behaviors and cognitive functions rely on an optimal balance between functional integration and functional differentiation in cortical circuits, otherwise defined as brain complexity (Tononi and Edelman 1998; Sporns et al. 2000; Bassett and Bullmore 2009;
Deco et al. 2015). Neurophysiologically, this balance is contingent on the ability of multiple, functionally specialized groups of cortical neurons (differentiation) to engage in rapid causal interactions (integration) and produce complex patterns of activity.

Different metrics have been devised to gauge brain complexity based on the analysis of spontaneous activity in terms 
of entropy and mutual information (Tononi et al. 1994; Amigo et al. 2015), causal density (Seth et al. 2011), or through the integration of neuroimaging data in graph theoretical models (Bullmore and Sporns 2009; Deco et al. 2015). Recently, these metrics have been complemented by a novel measure of brain complexity in humans that is based on direct cortical perturbations: the Perturbational Complexity Index (PCI) (Casali et al. 2013; Koch et al. 2016). Measuring PCI involves 2 steps: (1) perturbing a subset of cortical neurons with transcranial magnetic stimulation (TMS) to engage distributed, causal interactions in the brain (integration) and (2) measuring the compressibility (algorithmic complexity) of the resulting electroencephalographic (EEG) response.

Empirically, it has been demonstrated that the complexity of cortical responses (i.e., PCI) reliably discriminates between consciousness and unconsciousness in healthy humans during sleep and anesthesia as well as in brain-injured patients. Indeed, when consciousness is lost during non-rapid eye movement (NREM) sleep (Massimini et al. 2005), anesthesia (Ferrarelli et al. 2010), and the vegetative state (Rosanova et al. 2012), the large-scale differentiated pattern of cortical activation usually triggered by TMS during wakefulness invariably collapses into a low-complexity slow wave. Crucially, perturbational complexity is recovered upon awakening from NREM sleep or anesthesia and when patients recover consciousness from the vegetative state (Rosanova et al. 2012). In all these cases, the TMS-evoked slow wave typical of unconscious states is replaced by a complex spatiotemporal response with high PCI. These bedside TMS/EEG measurements are clinically relevant (Casarotto et al. 2016) and raise the general question of which fundamental mechanisms underlie the reduction and the recovery of complex responses in cortical circuits.

Intracranial electrical stimulations (ESs) and recordings in humans (Pigorini et al. 2015) suggest that, at least during NREM sleep, the slow-wave-like response evoked by direct perturbation might be reflective of an underlying network bistability, that is, the tendency of cortical neurons to fall into a silent period or Down state after an initial activation (Sanchez-Vives and Mattia 2014). An interesting possibility is that such bistable dynamics prevents causal interactions between distributed groups of cortical neurons (Pigorini et al. 2015), ultimately leading to the loss of complexity observed during sleep, anesthesia, and pathological conditions in humans (Massimini et al. 2012).

Thoroughly exploring and understanding the relationships between bistability, causality, and complexity in cortical networks requires a simplified model. To this end, in vitro cortical slices represent a good option for different reasons: (1) they are accessible to direct ES and multielectrode recordings; (2) they have been extensively validated as a model for cortical bistability through the emergence of spontaneous sleep-like slow oscillations (SOs) (Sanchez-Vives and McCormick 2000); (3) they reproduce the fundamental behavior of cortical circuits after deafferentation (Timofeev et al. 2000), a common consequence of brain injury; and (4) the emergent activity can be manipulated by different means, such as pharmacological agents.

Here, we test whether the ability of cortical circuits to engage in complex patterns of causal interactions can be enhanced by reducing sleep-like bistability or by manipulating excitability in cortical neurons. To this end we employed 2D arrays to record the response of cortical slices to direct ES performed in different conditions: (1) during a pattern characterized by sleep-like bistability reflected in the emergent SOs, (2) in an "awake-like" state where neuronal bistability is reduced by bath application of norepinephrine (NE) and carbachol (CCh), drugs with effects largely mediated through blockade of $\mathrm{K}^{+}$channels (Foehring et al. 1989; Schwindt et al. 1989), and (3) after bath application of kainate (Ka), which increases excitability of the cortical network (Cunningham et al. 2003). We then measured the ability of the networks to sustain a deterministic pattern of activation by means of phase-locking analysis and we computed the complexity of multisite cortical responses by developing an in vitro adapted version of PCI (henceforth, slice PCI or SPCI).

We found that bistability in cortical slices is associated with an early break-off of phase-locking of the response and with low-sPCI values. A reduction of bistability in awake-like states resulted in sustained phase-locking and higher SPCI values, a result that was not the consequence of merely increasing excitability. These findings show that cortical complexity can be neuromodulated in vitro and provide a valid model to understand the mechanisms of loss and recovery of complexity as measured by means of TMS/EEG in healthy and pathological human brains.

\section{Materials and Methods}

\section{Slice Preparation and Pharmacological Manipulation}

Ferrets were cared for and treated in accordance with Spanish regulatory laws (BOE 256; 25 October 1990), which comply with the European Union guidelines on protection of vertebrates used for experimentation (Strasburg 18 March 1986). All experiments were approved by the Ethics Committee of the Hospital Clinic.

Slices were prepared as previously described (Sanchez-Vives 2012). Briefly, animals (4-7 months old, either sex) were anesthetized with sodium pentobarbital $(40 \mathrm{mg} / \mathrm{kg})$ and decapitated. The entire forebrain was rapidly removed and placed in oxygenated cold $\left(4-10^{\circ} \mathrm{C}\right)$ bathing medium. $400-\mu \mathrm{m}$-thick coronal slices of the occipital cortex containing primary and secondary visual cortical areas (areas 17, 18, and 19) were used. A modification of the sucrose-substitution technique was used during the preparation to increase tissue viability (Aghajanian and Rasmussen 1989).

Slices were then placed in an interface style recording chamber and bathed for $15 \mathrm{~min}$ in an equal mixture of the sucrosesubstituted solution and artificial cerebrospinal fluid (ACSF). Afterwards slices were maintained $1-2 \mathrm{~h}$ in ACSF for recovery. Throughout the rest of the experiment an in vivo-like ACSF was applied. Solutions were aerated with $95 \% \mathrm{O}_{2}, 5 \% \mathrm{CO}_{2}$ to a final $\mathrm{pH}$ of 7.4, and the temperature during the experiment was maintained at $34.5-36^{\circ} \mathrm{C}$. ACSF contained (in $\mathrm{mM}$ ): $\mathrm{NaCl}, 126 ; \mathrm{KCl}, 2.5 ; \mathrm{MgSO}_{4}, 2$; $\mathrm{NaH}_{2} \mathrm{PO}_{4}, 1 ; \mathrm{CaCl}_{2}, 2 ; \mathrm{NaHCO}_{3}, 26$; and dextrose, 10. The modified ACSF had the same ionic composition except for different levels of (in $\mathrm{mM}$ ): $\mathrm{KCl}, 4 ; \mathrm{MgSO}_{4}, 1$; and $\mathrm{CaCl}_{2}$, 1. Electrophysiological recordings started after allowing at least $2 \mathrm{~h}$ of recovery.

Pharmacological manipulation was done by dissolving drugs in the ACSF: CCh (Sigma-Aldrich) and NE (Sigma-Aldrich) were applied at a concentration of 0.5 and $50 \mu \mathrm{M}$ respectively, while $\mathrm{Ka}$ (Tocris) was applied at a concentration of $0.2 \mu \mathrm{M}$. We usually waited more than $2000 \mathrm{~s}$ after the application of the drugs in order to let them act and to obtain a stable pattern of electrical activity.

\section{Electrophysiological Recordings}

Extracellular local field potentials (LFPs) were obtained with a 16-channel SU-8-based flexible microprobe (Illa et al. 2015) with a spatial distribution spanning both infra- (8 channels) and supra-granular (8 channels) layers (see Fig. 1a). The raw signal was amplified by 100 and high-pass filtered (cut frequency $0.1 \mathrm{~Hz}$ ) (Multichannel System MCS GmbH-Harvard Bioscience 
(a)

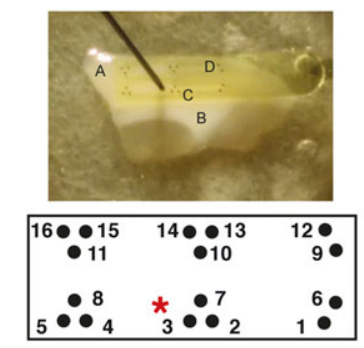

(e)

(f)

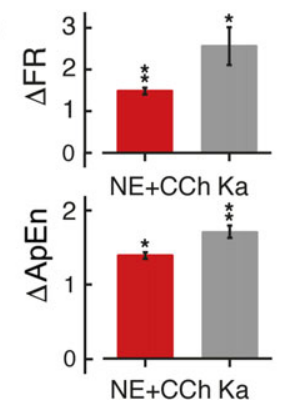

(b)

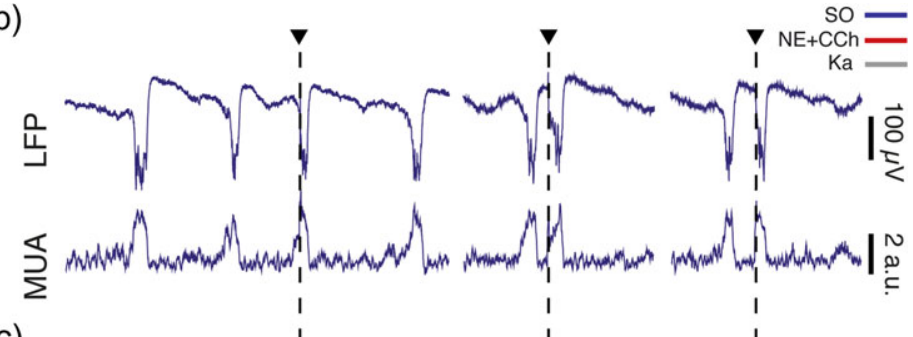

(c)

(d)

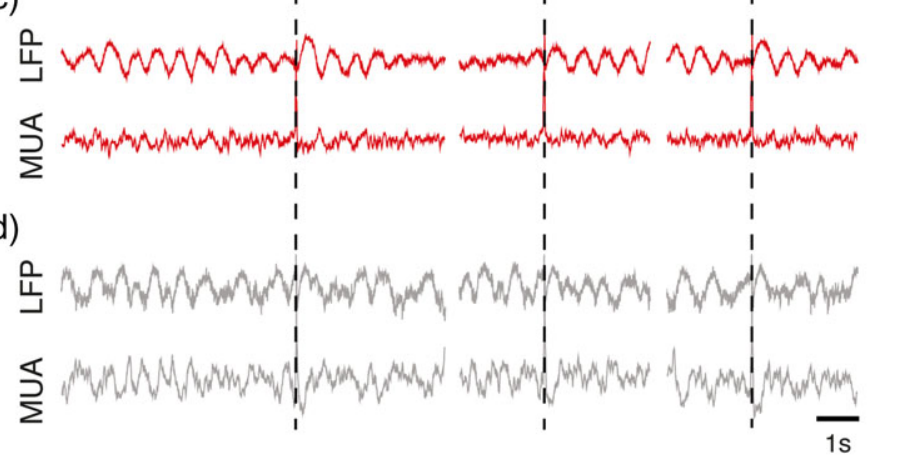

Figure 1. Cortical slice recording setup and experimental conditions. (a) Setup arrangement of recording and stimulating electrodes on a V1 ferret coronal slice including cortex (A) and white matter (B). The recording array was placed on the cortex, with 8 out of 16 recording sites (C) covering deep layers (numbers from 1 to 8 in the electrode scheme), and the remaining 8 spanning superficial layers (D) (from 9 to 16 in the scheme). Stimulation was carried out through a concentric electrode placed next to recording sites 3 and 7 (red asterisk in the scheme), reaching the cortex through a hole in the recording array. (b) Three fragments of spontaneous emerging activity in the slice, LFP (top) and MUA (bottom) in the control condition (SOs, blue). Black triangles at the top and discontinuous lines indicate moments of ES. (c) Same in $50 \mu \mathrm{M} \mathrm{NE}$ and $0.5 \mu \mathrm{M}$ CCh, red. (d) Same in $0.2 \mu \mathrm{M}$ kainic acid, gray. (e) Normalized increase in firing rate following the application of NE + CCh (red) and of Ka (gray) with respect to the control (SOs) condition. Average across slices and channels. (f) Same for entropy measurements.

Inc, Reutlingen, Germany) and digitized at $10 \mathrm{kHz}$ (Power1401 analog-to-digital converter/digital-to-analog converter (ADC/ DAC), Cambridge Electronic Design).

\section{Electrical Stimulation}

Single pulse ES was applied using concentric electrodes $(125 \mu \mathrm{m}$ diameter; FHC) placed in infragranular layers close to recording sites 3 and 7 as illustrated in Fig. 1a. The site of stimulation (infragranular or deep layers) was chosen based on the notion that spontaneous Up states are initiated in layer 5 (Sanchez-Vives and McCormick 2000; Chauvette et al. 2009; Sakata and Harris 2009). Pulses had a duration of $0.1 \mathrm{~ms}$, an intensity of $150 \mu \mathrm{A}$, and were applied every $10 \mathrm{~s}$, with a random jitter of $0.5-1.5 \mathrm{~s}$ to avoid activity entrainment to the specific frequency of stimulation. Such parameter selection was based on preliminary tests in order to assure consistency and repeatability of the responses. In particular, we aimed at obtaining a complete SO cycle in response to each pulse when the network was showing spontaneous SOs (Shu et al. 2003). This assured in turn consistency in the responses to electric pulses in the conditions of higher excitability (NE + CCh, Ka). The stimulation was triggered from Spike2 using a Power1401 ADC/ DAC (Cambridge Electronic Design) and converted to a current pulse using a stimulus isolator (360A, WPI). For each of the three conditions (SO, $\mathrm{NE}+\mathrm{CCh}, \mathrm{Ka}$ ), we recorded $50 \mathrm{~s}$ of spontaneous activity before starting the stimulation phase, which lasted for 160 s such that the number of ESs applied was 15.

\section{Data Preprocessing, Multiunit Activity, and SO Parameters}

Analysis was performed with custom software written in Matlab (Mathworks). Multiunit activity (MUA) was estimated as previously described (Reig et al. 2010): given that power spectra of population firing rate have Fourier components proportional to the firing rate itself (Mattia and Del Giudice 2002), highfrequency components of the LFP can be seen as a linear transform of spiking activity. Thus, power changes in the Fourier components at high frequencies of the LFP provide a reliable estimation of the population firing rate. MUA traces were calculated then as the average power of the normalized spectra in the frequency band $0.2-1.5 \mathrm{kHz}$, and downsampled at $200 \mathrm{~Hz}$. To balance large fluctuations of nearby spikes, MUAs were scaled logarithmically. Furthermore, a moving average with a sliding window of $80 \mathrm{~ms}$ was applied to smooth the MUA time series. The MUA waveforms (or firing rate) cited in the main text refer to these filtered logarithmic traces. Upward and downward transitions to identify Up states were individuated by posing a time and an amplitude threshold on the smoothed MUA traces. Up state frequency of a recording period was then defined as the inverse of the mean interval between consecutive Up state onsets.

To analyze the response to stimulation, MUA waveforms of every trial were aligned at the stimulation time, and a range starting $1 \mathrm{~s}$ before the pulse and ending $2 \mathrm{~s}$ after was taken into account. The total amount of firing was calculated by adding up MUA values for each time sample across channels, subtracting baseline (averaged value of the prestimulus activity) and then averaging across slices.

\section{Relative Power Spectrum and Approximated Entropy}

Power spectrum density analysis was carried out by Welch's method with Hanning windowing on 50 s of spontaneous activity. For each channel and slice, the values in the range $(200-1500 \mathrm{~Hz})$ of the obtained spectra in $\mathrm{NE}+\mathrm{Cch}$ and $\mathrm{Ka}$ 
conditions were added up and then normalized to each respective measure during SO.

Approximated Entropy (ApEn) was computed to measure signal irregularity based on how frequently individual temporal patterns reoccur in the data (Pincus and Goldberger 1994; Schmidt et al. 2013), and was obtained using a script for Matlab written by Kijoon Lee based on (Chon et al. 2009). Briefly, the algorithm scans the data looking for approximated matches for detected patterns of $30 \mathrm{~ms}$ of length, imposing a tolerance threshold on similarity of $0.5 \times$ std of the analyzed data (both parameters were chosen as the ones that maximized the entropy measure (Chon et al. 2009)). For each of these patterns, all matches are evaluated considering the first subsequent point: if this data point is different, ApEn is increased. ApEn was computed separately for each channel and slice on $50 \mathrm{~s}$ of spontaneous MUA traces, after low-pass filtering (cut-off frequency $2 \mathrm{~Hz}$ ) to capture regularity of firing rate changes at low frequencies.

\section{Phase-Locking Analysis}

Instantaneous phase-locking factor (PLF) was computed as reported in (Sinkkonen et al. 1995). Briefly, PLF is obtained as the absolute value of the average of the Hilbert Transform of MUA responses for all single trials. Statistical differences from baseline were assessed for each channel by using a bootstrap procedure: for each channel, prestimulus points (from -1 to $-0.5 \mathrm{~s}$ with respect to the stimulation) were random shuffled (Lv et al. 2007) and the instantaneous maximum value across such surrogated data set was computed. This procedure was repeated 350 times to obtain a distribution of 35000 bootstraps (350 permutations $\times$ 100 samples). The 1-tail (1- $\alpha$ ) 100th percentile of this distribution was used to estimate a significance threshold for the centered averaged data for that channel and those PLF values below threshold were set to zero. Then, in order to eliminate spurious significant points, values over the threshold that did not last for at least 3 consecutive time stamps (i.e., for at least $15 \mathrm{~ms}$ ) in at least 3 channels, were also set to 0 . Comparisons between slices and conditions were computed by averaging across slices the mean maximum time in which PLF was significant across channels (i.e. maximum time of significant PLF or max PLFt).

\section{Adaptation of PCI Algorithm for In Vitro Recordings}

The PCI was computed with an adapted version for in vitro recordings of the original algorithm (Casali et al. 2013) describing EEG responses in humans to TMS stimulation. The main differences are on the data preprocessing. In the original algorithm, Current Source Density Analysis was performed on EEG recordings to avoid effects of volume conduction, and processed data were then centralized. As the number of sources was very high (about 3000), data were normalized with the standard score method in order to have a robust procedure for multiple comparisons. In the case of in vitro LFP recordings volume conduction is negligible, thus we decided to calculate PCI directly from the recorded signal. SPCI was calculated from the MUA traces since the LFP is often subject to noise originating from many sources, such as fluctuations in the solution flux, electrical power, and because MUA is directly tight to neuronal activity. Furthermore, the number of channels in our case was much lower (16), so we switched from a global to a local statistics procedure, and channels were analyzed independently. Data centralization was made by subtracting for each channel the mean prestimulus activity. Statistical comparison was performed through the same bootstrap procedure used for phase-locking analysis, but in this case surrogate averaged waveforms were centralized. The final bootstrap distribution was constructed out of the instantaneous mean values of the surrogate data set (instead of the maximum), and finally composed by 70000 points as we took into account the entire $1 \mathrm{~s}$ preceding the stimuli. In this way, a binary spatiotemporal distribution of significant activity $(\mathbf{S S}(x, t))$ was calculated: $\mathbf{S S}(x, t)=1$ for significant activity at channel $x$ and time $t$, SS $(x, t)=0$ otherwise (Casali et al. 2013). Statistical significant level $\alpha$ was set to $<0.001$ to have very conservative statistics.

The remaining part of the in vitro SPCI algorithm is identical to the original one as explained in detail in (Casali et al. 2013). Briefly, Lempel-Ziv complexity $\left(c_{\mathrm{L}}\right)$ was computed on the SS $(x, t)$ binary matrices of dimension $L$ (total number of spatiotemporal samples $=$ number of channels $\times$ number of samples), using an adapted routine from (Kaspar and Schuster 1987). The algorithm ran through the first columns of the input matrix searching for patterns, and this search was repeated for each subsequent column while keeping track of patterns encountered in previous columns. The asymptotic behavior of $c_{L}$ for random strings is $\mathrm{LH}(\mathrm{L}) / \log _{2}(\mathrm{~L})$, where $\mathrm{H}(\mathrm{L})$ is the source entropy:

$$
H(L)=-p_{1} \log _{2}\left(p_{1}\right)-\left(1-p_{1}\right) \log _{2}\left(1-p_{1}\right)
$$

and $p_{1}$ is the fraction of " 1 " contained in the binary string of length $\mathrm{L}$.

The sPCI was then defined as the normalized Lempel-Ziv complexity $\left(\bar{c}_{\mathrm{L}}\right)$ of the evoked MUA spatiotemporal patterns, SS $(x, t)$, with $x=1 \ldots L_{1}$ and $t=1 \ldots L_{2}$. Channels were sorted by their number of significant samples, as an approximation of the equalization with the lower complexity of the spatial ordination of the binary matrices.

Having these matrices as input, for each sample the algorithm calculated a number $c_{1}(t)$ that corresponded to the Lempel-Ziv complexity of the bi-dimensional sequence of length $l(t)$. sPCI was then equated to the Lempel-Ziv complexity of the complete bi-dimensional sequence of length $L=L_{1} \times L_{2}$, normalized by $L H(L) / \log _{2}(L)$ :

$$
\mathrm{SPCI}=c_{l}\left(t=L_{2}\right) \times \frac{\log _{2}(L)}{L H(L)} .
$$

The normalization on the source entropy assures that the complexity measure is minimally dependent on the total amount of significant activity, and maximally dependent on the formation of patterns in the data.

Furthermore, the time evolution of SPCI can be studied by computing the index for intervals of increasing duration:

$$
\operatorname{sPCI}(t)=c_{l}(t) \times \frac{\log _{2}(L)}{L H(L)} .
$$

\section{Other Analyses and Statistics}

The onset of the Down state evoked during SOs (Fig. 3c) was identified by searching in each slice and channel for strong positive deflections in the averaged LFP signal and a correspondent fast suppression of the firing rate in the MUA waveforms. A supervised automatic procedure was applied to detect the transition from the Up to the Down state, which was identified as the moment when the MUA was sustained for a minimum of $50 \mathrm{~ms}$ and then dropped $10 \%$ below the baseline activity. The correlation of those values with the max PLFt measure was described with multiple linear regressions. Outlier values were individuated by imposing a threshold of 10 standard deviations 
from the data median, and excluded from the regressions. Before fitting, data were grouped by computing the agglomerative hierarchical cluster tree (Matlab "linkage" function), using the Weighted Average Distance method, with first-order Minkowski metrics. Then clusters were constructed from the tree by imposing 4 as the maximum number of clusters (Matlab "cluster" function). Sixty points (light-gray cluster in Fig. 3c) were excluded from the fit since they corresponded to those channels in which stimulation was not effective. While such condition created a bias in the recognition of the Down state onset, it did not affect the computation of PLF or of the PCI. The statistics in both procedures assured that parts of the responses not reproducible across ESs did not result in false phase-locking or significant points in the binary matrices from which the PCI is calculated (see below).

Significant changes in the firing rate and ApEn for the NE + $\mathrm{CCh}$ and $\mathrm{Ka}$ conditions with respect to the SOs condition were evaluated with 1-sample t-tests, while significant changes in PLFt and sPCI populations to compare the NE + CCh and $\mathrm{Ka}$ conditions with the SOs condition were evaluated with the 2sample Kolmogorov-Smirnov test $(\alpha=0.01)$.

\section{Results}

Extracellular LFPs were recorded from a total of 29 ferret acute primary visual cortex (V1) coronal slices (Sanchez-Vives and McCormick 2000) initially engaged in the generation of Up and Down states or SOs (Steriade et al. 1993; Sanchez-Vives and McCormick 2000; Compte et al. 2008). An additional sample ( $n=$ 21) including recordings from other cortical areas (prefrontal, motor, and somatosensory cortex) is reported in the Supplementary Data. We recorded cortical activity by means of 16-channel arrays covering an area of $3 \mathrm{~mm}$ by $0.866 \mathrm{~mm}$. Eight channels were used to record from the upper half of the cortex and eight channels from the lower half, corresponding to superficial versus deep layers, respectively (Fig. 1a). ES was delivered through bipolar electrodes in the form of single pulses applied to layer 5 (Fig. 1a). The stimulation parameters (intensity $150 \mu \mathrm{A}$, duration $0.1 \mathrm{~ms}, 15$ trials, interval $10 \mathrm{~s} \pm$ a random jitter between [0.5-1.5] s) were empirically selected based on preliminary experimental trials in order to assure consistency and reliability of the responses. From the LFP, we computed the MUA. The MUA provides a reliable estimation of the local population firing rate (Mattia and Del Giudice 2002; Reig et al. 2010). The responses to the ES were observed under 3 different conditions: during SOs, in an "awake-like" state in the presence of bath-applied NE (Constantinople and Bruno 2011) and CCh, and in a condition of increased network excitability induced by the bath application of $\mathrm{Ka}$ (Cunningham et al. 2003). For more detailed information see the "Materials and Methods" section. The values in the following sections will be presented as "mean \pm SEM."

\section{Intrinsic Bistable Dynamics: Low Causality and Low Complexity}

First, we recorded spontaneous SOs generated by the visual cortex in vitro without pharmacological treatment (Sanchez-Vives and McCormick 2000). The emergent SOs were characterized by a mean frequency of $0.23 \pm 0.04 \mathrm{~Hz}(n=29)$. The active periods of the oscillation, or Up states, were identified by negative deflections in the LFP traces and by an increase in the MUA for a mean duration of $0.30 \pm 0.03 \mathrm{~s}$. Up states alternated with silent periods, or Down states, that lasted on average $4.76 \pm 0.70 \mathrm{~s}$ and where the
LFP underwent a positive deflection and the firing rate was low (Fig. 1b).

In this network state, the electric pulses delivered randomly to layer 5 invariably evoked a SO (Shu et al. 2003) (Fig. 1b). Evoked Up states were highly similar to the spontaneous ones (Shu et al. 2003) and reproducible across trials (Fig. 2a, $n=15$ ). The evoked Up states were characterized by a strong negative deflection in the LFP (\#1 in Fig. 2a) and were associated with an increased level of firing visible in the MUA trace (Fig. 2b). This period of activation was followed by a long-lasting Down state, characterized by a positive deflection in the LFP (\#2 in Fig. 2a) and by cessation of firing. Thus, following a stimulus, the network engaged in the generation of a slow wave, revealing its own bistable dynamics in response to electrical stimuli. After this stereotyped bistable response, the neuronal activity gradually returned to the prestimulus level (\#3 in Fig. 2a).

Next, we investigated the impact of such intrinsic bistable dynamics on the deterministic effect of the perturbation and the overall network complexity. To this end, we first subtracted the mean prestimulus activity in each channel from the

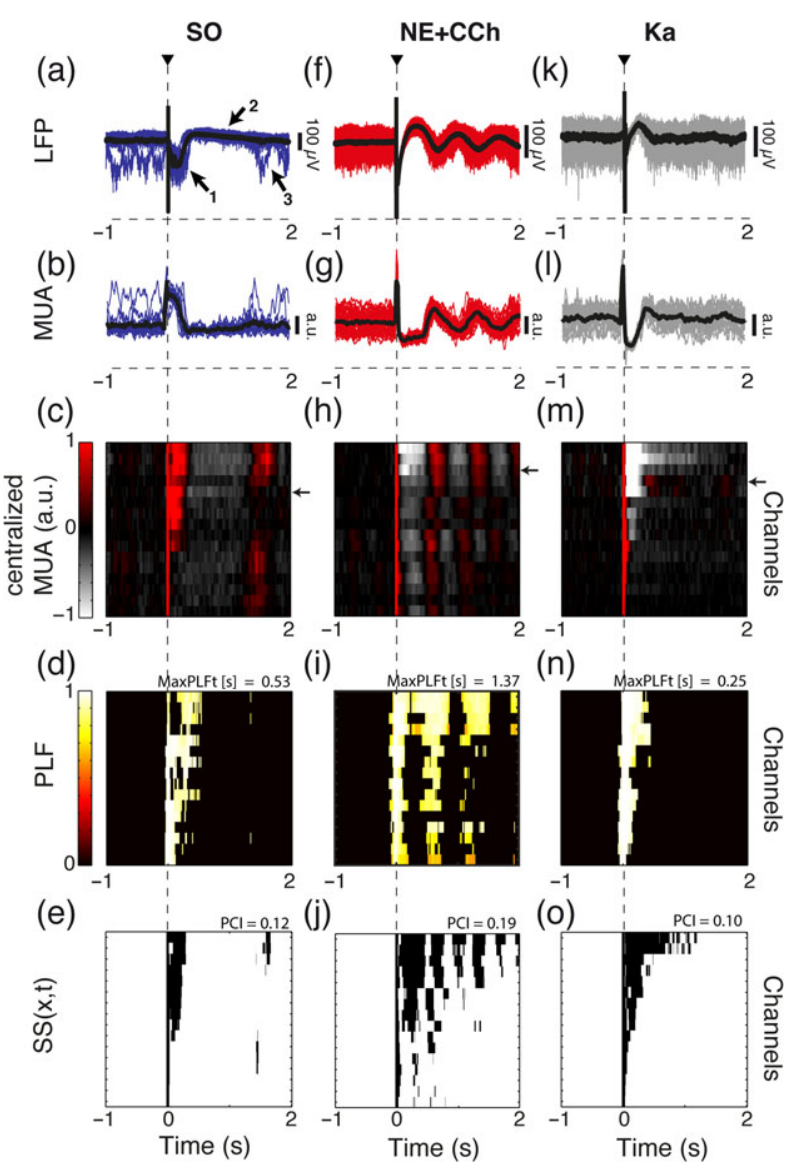

Figure 2. PLF and PCI calculated in three representative visual cortex slices during SOs, NE + CCh and $\mathrm{Ka}$. In (a) and (b) blue traces represent respectively LFP and MUA of all single $(n=15)$ trials obtained during a SO representative recording from one single channel. Numbers from 1 to 3 in the LFP panel represent the evoked Up state, the subsequent Down state and the recovery of spontaneous activity, respectively. Black lines indicate the average. For all channels of the same slice (black arrow on the right), panels $c, d$, and $e$ show centralized averaged MUA, statistically significant PLF and binary statistics matrices of significant activity, respectively. In all these cases, channels are sorted by the total amount of statistically significant activity. $f-j$. Same for a representative $\mathrm{NE}+$ CCh recording $(n=15)$. $k-0$. Same for a representative Ka recording $(n=15)$. 
average MUA responses (centralization, see "Materials and Methods") to emphasize the departure from the baseline and to allow comparison across trials. An example of the result of this procedure is depicted in Fig. 2c, which shows the centralized MUA obtained from all the channels recorded in a representative slice for the SO condition. Then, based on the MUA, we assessed the deterministic effects of the stimulation by computing the instantaneous PLF (Pigorini et al. 2015), defined as the absolute value of the mean Hilbert Transform of the single trial responses. A bootstrap procedure was employed to identify when the instantaneous PLF was significantly different from the baseline and all values below the threshold $(\alpha<0.001)$ were set to zero (see "Materials and Methods"). Figure $2 d$ shows the time course of the significant PLF for all channels of a slice in the SO condition.

Finally, in order to quantify the complexity of the spatiotemporal response induced by ES, we computed the SPCI by using a procedure adapted from the one originally used to calculate the PCI from TMS-evoked cortical activations in humans (Casali et al. 2013). Here, the spatiotemporal response was represented by the statistically significant poststimulus activity of the centralized MUA for each time sample and channel, with respect to the prestimulus activity. Significant increases and decreases of MUA activity with respect to baseline were then set to 1 whereas nonsignificant variations were set to 0 , resulting in a binary matrix. By analogy with the matrices of TMSevoked cortical significant sources obtained in humans, these matrices of electrically evoked significant MUA are called $\mathbf{S S}(x, t)$ (Fig. 2e). As in the original PCI calculation, the Lempel-Ziv complexity was then computed on the SS $(x, t)$ binary matrix of spatiotemporal activation and the result was normalized by the source entropy, thus obtaining sPCI (see "Materials and Methods"). In the SO condition $(n=29)$, the deterministic component of the response was limited to the initial activation and was soon obliterated by the occurrence of a long-lasting Down state followed by a stochastic resumption of MUA. Thus, the SS $(x, t)$ binary matrix was restricted and easily compressible (Fig. 2e).

\section{Reducing Bistability Increases Causality and Complexity}

We next simulated the neuromodulatory activating system (Foehring et al. 1989; Schwindt et al. 1989) by means of bath application of noradrenergic $(50 \mu \mathrm{M} \mathrm{NE})$ and cholinergic $(0.5 \mu \mathrm{M}$ $\mathrm{CCh}$ ) agonists to 14 visual cortex slices and to 21 slices from other cortical areas: prefrontal $(n=11)$, somatosensory $(n=5)$, and motor cortex $(n=5)$ (see Supplementary Data). We report here the results from the visual cortex slices. The concentration of these neuromodulators varies with arousal (Jones 2004) and they are known to affect cortical dynamics (Constantinople and Bruno 2011) by reducing network bistability consisting of Up and Down states. Following the application of NE + CCh, the slow, regular alternation between Up and Down states was replaced by irregular fluctuations of the LFP at around $2-3 \mathrm{~Hz}$ (Fig. 1c), on a background of enhanced high-frequency (beta, gamma) activity. The population firing rate was increased on average by $1.48 \pm 0.08$ (Fig. $1 e, P=3.98 \times 10^{-8}$, 1 -sample t-test) with respect to the firing observed during the Up states of the SO condition. To further compare the "awake-like" state with the departing SO condition, we calculated an index of pattern regularity, the ApEn (Pincus and Goldberger 1994) computed for the spontaneous MUA patterns recorded through each channel. In the presence of $\mathrm{NE}+\mathrm{CCh}$, the ApEn increased by $1.39 \pm 0.04$
(Fig. 1f, $\mathrm{P}=1-50 \times 10^{-13}, 1$-sample t-test) with respect to the SO condition (Schmidt et al. 2013).

$\mathrm{NE}+\mathrm{CCh}$ induced clear-cut changes in the slice responses to electrical perturbation in the LFP (Fig. 2f). The activation produced by the ES led to a sharp response followed by a shortlasting $(<0.25 \mathrm{~s})$ decrease in firing rates $(-1.15 \pm 0.04 \mathrm{a}$.u. with respect to the prestimulus baseline in the representative channel in Fig. 2g), which was followed by a rebound of neuronal activity. This resumption of neuronal firing had a temporal structure, including a chain of responses activated at different latencies (Fig. 2h. Frequency $1.47 \mathrm{~Hz}$ for the representative channel in Fig. 2f,g) that recurred for up to $2 \mathrm{~s}$.

The PLF duration increased substantially in NE + CCh (up to $2 \mathrm{~s}$ ) with respect to the SO condition (Fig. 2i). In this case, the SS $(x, t)$ was extended in space and time (Fig. $2 j)$ throughout the sequence of significant decreases and increases of MUA evoked by the stimulation and thus the SPCI attained higher values.

\section{Increases in Causality and Complexity Cannot be Explained in Full by Enhanced Excitability}

Finally, we asked whether the increase in complexity and phase-locking duration observed during NE + CCh administration could be simply due to increased excitability in the network. To address this question, we applied $0.2 \mu \mathrm{M} \mathrm{Ka}$ in the bath to 15 visual cortex slices that presented spontaneous SOs. Ka is an ionotropic glutamatergic receptor agonist that has often been used to enhance excitability; it is known to induce a broadband power increase in the activity with emerging peaks in the beta and gamma ranges (Cunningham et al. 2003), probably following enhanced spiking activity (Ray et al. 2008). Similar to the NE + CCh condition, the application of $0.2 \mu \mathrm{M} \mathrm{Ka}$ resulted in an irregular pattern characterized by a dominant $2-3 \mathrm{~Hz}$ rhythm (Fig. 1d) and enhanced high-frequency oscillations (beta and gamma) in the LFP (not shown). Consistently, the population firing rate increased by a factor of $2.56 \pm 0.45$ with respect to the one detected during the Up states of the SO (Fig. $1 e, P=7.27 \times 10^{-4}$, 1-sample t-test), thus enhancing excitability to a greater extent than in the $\mathrm{NE}+\mathrm{CCh}$ condition $(P=0.041$, 2-sample Kolmogorov-Smirnov test). Further strengthening this notion, we calculated the ApEn (Pincus and Goldberger 1994). In the presence of $\mathrm{Ka}$, the ApEn increased by a factor of $1.71 \pm 0.08$ with respect to the SO condition (Schmidt et al. 2013) (Fig. 1f, $P=$ $7.44 \times 10^{-17}$, 1-sample t-test). Ka, therefore, increased the ApEn of the population firing rate significantly more than $\mathrm{NE}+\mathrm{CCh}$ did $\left(P=6.76 \times 10^{-4}, 2\right.$-sample Kolmogorov-Smirnov test). Despite such increased excitability, the ES during Ka triggered a simple LFP response (Fig. $2 k$ ). The response was associated with a sharp activation followed by a brief silent period $(-1.38 \pm 0.02$ a.u. with respect to the prestimulus baseline in the MUA trace of the representative channel in Fig. 2l). This was followed by a prompt resumption of firing (Fig. 2l,m). Crucially, the rebound of activity observed during Ka was not time-locked to the stimulation and lacked an organized sequence of evoked waves like the one observed in the NE + CCh condition. Hence, kainic acid produced a stronger enhancement of network excitability (as reflected in the firing rate and in the entropy measures), but failed to increase the PLF duration (Fig. 2n), and SS(x,t) (Fig. 20) resulting in low-sPCI values in the visual cortex.

To investigate whether these observations could extend to other neocortical areas, we applied $0.2 \mu \mathrm{M} \mathrm{Ka}$ to an additional sample of 21 slices: prefrontal $(n=11)$, somatosensory $(n=5)$, and motor cortex $(n=5)$. In this more heterogeneous sample, $\mathrm{Ka}$ induced a significant increase in SPCI with respect to 
control; however, this increase was significantly smaller than the one induced by NE + CCh (see Supplementary Data Figs 1 and 3). This finding points to a certain dependence of sPCI on excitability, which is negligible in the visual cortex but more evident in other areas of the neocortex. Nevertheless, no relationship was found between the firing rate and the SPCI within conditions (see Supplementary Data Fig. 2).

\section{Phase-Locking and Complexity of Cortical Responses Across Conditions}

To systematically assess significant differences across conditions, we computed, for each visual cortex slice, the average duration of the significant PLF across all channels (max PLFt, Fig. 3a). Despite inter-channel variability in the intensity of the response, the values of max PLFt in the SOs condition (blue histogram) were narrowly distributed with an average max PLFt of $0.25 \pm 0.02 \mathrm{~s}(n=29$, Fig. $3 b)$, roughly corresponding to the duration of the evoked Up state. Interestingly, in the SO condition we found that the time of decay of PLF was linearly correlated with the onset of the Down state (see "Materials and Methods"), shown for each channel and slice in Fig. $3 c(P=1.54$ $\times 10^{-78}$, multiple linear regression between black and green points, black line). Sixty points (out of 464 ; light-gray cluster in
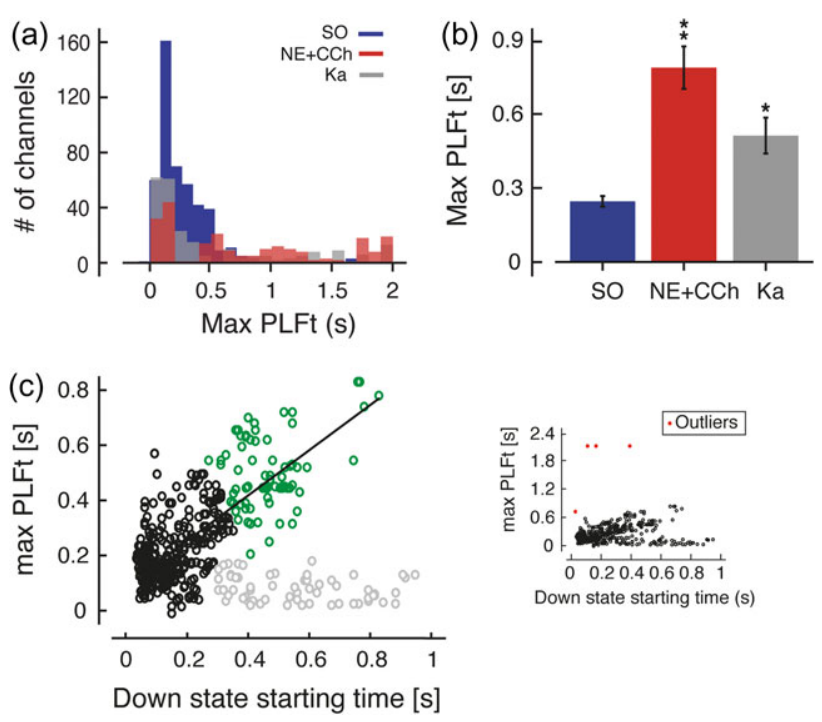

Figure 3. Population PLF of responses and its relationship to the initiation of the Down state. (a) Distribution of max PLFt (maximum time of significant PLF) values for each channel and slice in the SO condition (blue, $n=464$ corresponding to 16 channels for 29 slices), in the NE + CCh condition (red, $n=224$ corresponding to 16 channels for 14 visual cortex slices) and in the Ka condition (gray, $n=240$ corresponding to 16 channels for 15 slices). (b) Average max PLFt across channels and slices averaged from the data in a $\left({ }^{*} \mathrm{P}=8.90 \times 10^{-3} ;{ }^{* *} \mathrm{P}=\right.$ $4.38 \times 10^{-6}, 2$-sample Kolmogorov-Smirnov test). (c) Correlation of Down state starting time with max PLFt for each channel and slice during SOs (black and green points, $n=464$ corresponding to 16 channels for 29 slices). Values are reported with respect to the stimulation time. Four outliers (red dots in the inset on the right-hand side) were identified by posing a threshold of 10 STD of distance from the data median, and were excluded from the fit. Data were grouped by hierarchical clustering analysis, and then fitted with a linear regression taking into account 2 of the 3 obtained clusters $\left(P=1.54 \times 10^{-78}\right.$, multiple linear regression, black and green points, black line). Those 2 clusters represent channels with shorter lasting responses and long-lasting responses. Sixty points (light-gray cluster) were excluded from the fit since it was a clear-cut population with no relationship between the Down state initiation and max PLFt, corresponding to cases where the stimulation seldom evoked a SO (for more information check "Materials and Methods").
Fig. 3c) identified by hierarchical clustering analysis were excluded from the fit because of the inconsistency of their response to the stimulus (for details see caption of Fig. $3 c$ ).

In $\mathrm{NE}+\mathrm{CCh}$ the average max PLFt across channels and slices (Fig. 3a) was significantly prolonged by $0.79 \pm 0.09 \mathrm{~s}$ with respect to the SO condition (Fig. $3 b, P=4.38 \times 10^{-6}$, 2-sample KolmogorovSmirnov test). In $\mathrm{Ka}$, the average max PLFt across all slices was $0.51 \pm 0.07 \mathrm{~s}$, which was significantly longer when compared with the SO condition (Fig. $3 b$, Ka condition vs. SO condition: $P=8.90 \times$ $10^{-3}$, 2-sample Kolmogorov-Smirnov test), and with a trend towards being shorter than the one observed in NE + CCh (not statistically significant; 2-sample Kolmogorov-Smirnov test).

Finally, we computed and compared the values and the time course of sPCI for all channels and slices across the 3 conditions. Figure $4 a$ shows that, on average, sPCI values in the SO condition and in the $\mathrm{Ka}$ condition were not statistically different $(0.103 \pm$ 0.005 and $0.117 \pm 0.001$, respectively) and, in both cases, were significantly lower with respect to the NE + CCh condition (0.184 \pm $0.004, P=2.84 \times 10^{-7}, 2$-sample Kolmogorov-Smirnov test). Along the same line, the time courses of sPCI calculated across slices in the SO condition and in the Ka condition (respectively blue and gray line in Fig. $4 b$ ) were comparable, reaching the plateau $0.25 \mathrm{~s}$ after the stimulation, in spite of the differences in terms of firing rates and excitability between both conditions. In $\mathrm{NE}+\mathrm{CCh}$, instead, sPCI increased during the first $0.5 \mathrm{~s}$ after the stimulus and kept growing, albeit at a slower rate, up until $2 \mathrm{~s}$ (red line in Fig. 4b).

\section{Discussion}

\section{Bistability, Causality, and Complexity in Different Cortical Dynamic States}

A series of recent studies in humans have shown that quantifying the information content of the patterns of causal interactions induced by a direct cortical perturbation with TMS provides an index (PCI) that reliably correlates with the loss and recovery of consciousness in different conditions (Casali et al. 2013). These macroscale-level studies have practical implications at the bedside and raise the important question regarding the basic neuronal mechanisms responsible for the collapse and recovery of complexity as assessed through cortical perturbations. In the present work, we aimed at addressing this problem by applying a similar perturbational approach to cortical slices that were set to different functional states through pharmacological manipulations. At this microscale level, we found that: (1) during slow wave sleep-like dynamics (SO) the overall network complexity was low, (2) complexity in (a)

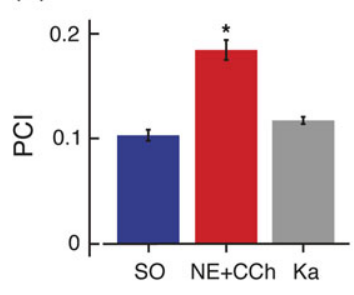

(b)

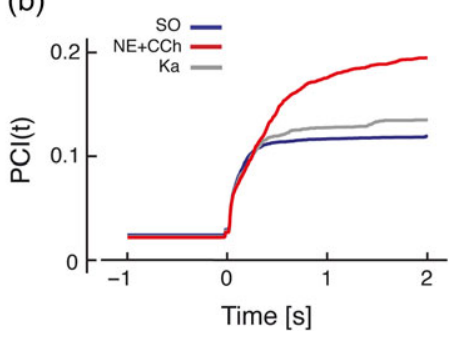

Figure 4. Population values and time evolution of slice PCI. (a) SPCI values averaged across visual cortex slices $(0.103 \pm 0.005$ for SO condition, $n=29 ; 0.184 \pm$ 0.004 for NE + CCh condition, $n=14, P=2.84 \times 10^{-7}$, 2-sample KolmogorovSmirnov test; $0.117 \pm 0.001$ for Ka condition, $n=15$ ). (b) Time evolution of sPCI in the 3 experimental conditions. 
the visual cortex was not increased by simply increasing neuronal excitability and firing rates (Ka), (3) the administration of noradrenergic and cholinergic agents ( $\mathrm{NE}+\mathrm{CCh}$ ), which reduce sleep-like bistability, was more effective in increasing perturbational complexity. In other neocortical areas (prefrontal, motor, and somatosensory cortices; Supplementary Data) we also observed a significant increase in $\mathrm{PCI}$ in $\mathrm{NE}+\mathrm{CCh}$ that returned to control values after their washout (see Supplementary Data Fig. 1). Differently from visual cortex, in those areas a significant increase in PCI in the Ka condition was also detected, albeit significantly smaller than that in the NE + CCh condition.

The baseline condition of our experiment was the default mode of activity typical of isolated cortical circuits in vitro (SanchezVives and McCormick 2000). In this condition, cortical networks display sleep-like spontaneous activity characterized by recurrent SOs. SOs are the hallmark of an intrinsic bistability between Up and Down states of cortical networks (Sanchez-Vives et al. 2017). The onset and maintenance of Up states is due to local reverberation in the cortex (Contreras et al. 1996; Sanchez-Vives and McCormick 2000; Compte et al. 2003; Mattia and Sanchez-Vives 2012), while the transition back to the Down state is thought to be controlled by a slow negative feedback given by slow $\mathrm{K}^{+}$currents (either $\mathrm{Ca}^{2+}$ - and $\mathrm{Na}^{+}$-dependent [Sanchez-Vives and McCormick 2000; Sanchez-Vives et al. 2010], or adenosine triphosphatedependent [Cunningham et al. 2006]), although other activitydependent mechanisms such as synaptic depression (Holcman and Tsodyks 2006) or $\mathrm{GABA}_{\mathrm{B}}$ activation (Mann et al. 2009) have also been suggested. The network fails to maintain the self-activated Up state when the build-up of activity-dependent $\mathrm{K}^{+}$currents overcomes the positive feedback inherent to local circuits (Compte et al. 2003). At this moment, the network falls into a silent state, and the persistence of the outward currents generates a prolonged hyperpolarization (Sanchez-Vives and McCormick 2000; Cunningham et al. 2006; Sanchez-Vives et al. 2010) that acts as a refractory period. Thus, due to bistability, cortical neurons tend to fall into a silent, hyperpolarized state (Down state) after an initial activation.

One interesting possibility is that bistability may prevent cortical circuits from engaging in sustained complex patterns of causal interactions. Indeed, when we applied local electrical stimuli to the slice in the baseline (SO) condition, we recorded an LFP characterized by an initial activation, similar to spontaneous Up states, followed by a silent Down state, after which MUA activity resumed with no relation to the stimulus, as demonstrated by phase-locking analysis. Thus, the Down state not only transiently interrupts neuronal firing but also disrupts the chain of deterministic effects brought about by the initial activation. In essence, the analysis of the MUA shows that cortical neurons, upon receiving an input (ES), following an initial activation, tend to hush and then forget. This observation is in line with previous studies showing that the resumption of neuronal firing after a spontaneously occurring Down state is a stochastic process (Sanchez-Vives and McCormick 2000; Compte et al. 2003; Luczak et al. 2007; Chauvette et al. 2011). Notably, in the SO condition, the onset of the evoked Down state coincided with both the timing of the drop of PLF (Fig. 3c) and the timing at which SPCI stopped growing (Fig $4 b$ ). This points to a mechanism by which bistability may lead to loss of complexity: (1) the bistable network falls into a silent Down state, (2) the Down state brings about an irreversible loss of causality, and (3) at this point, sPCI, which captures the information content of the deterministic network response, stops growing and only attains a low value (Figs 2 and 4).

Crucially, reducing bistability by adding NE and CCh to the bath prevented this process. NE $(1-100 \mu \mathrm{M})$ and CCh $(<1 \mu \mathrm{M})$ have been shown to increase spontaneous firing rate by a combination of a pronounced and instantaneous effect of the cholinergic agonist and a more moderated but outlasting effect of adrenergic neuromodulators (Schmidt et al. 2013), whose net effect is largely mediated by blocking $\mathrm{K}^{+}$conductances (Foehring et al. 1989; Schwindt et al. 1989). In this condition, the initial activation evoked a chain of deterministic causal interactions leading to high values of both PLF duration and sPCI. On the other hand, bistability could not be reduced by the application of $\mathrm{Ka}$, a glutamate agonist that is known to enhance excitability, high-frequency synchronization, and firing rates (Cunningham et al. 2003) but has a limited action on $\mathrm{K}^{+}$channels. Here, we observed that $\mathrm{Ka}$ in the visual cortex increased the firing rate (Fig.1e) and entropy (Fig.1f) of ongoing activity, but had no effects on causality and perturbational complexity (Figs 3 and 4). This observation is relevant for 2 reasons. First, it highlights a fundamental difference between spontaneous entropy and perturbational complexity; while the first may be maximal for random patterns that are not necessarily integrated, the latter captures only the share of information that is integrated through causal interactions. Second, it shows that increasing excitability is not enough to restore causality and complexity. In the additional sample from other cortical areas (prefrontal, motor, and somatosensory cortices reported in the Supplementary Data) Ka did induce a significant increase in PCI with respect to control, albeit signficantly smaller than that in NE + CCh. Still, no relationship was detected between firing rate and PCI for different slices and conditions (see Supplementary Data Fig. 2).

A certain dependence of PCI on excitability levels is expected based on the original formulation of this index (Casali et al., 2013): PCI measures the information content of brain responses that are integrated in space and time. Low levels of cortical excitability prevent the network from producing sustained responses and thus integration. Hence, a certain level of excitability may be necessary but not sufficient to attain high PCI. This principle is relevant for developing measures in humans, as there are different conditions, such as seizures, sleep, and some types of anesthesia, in which the cortex produces hyper-excitable, large responses that lack information content, or complexity (Massimini et al. 2007).

\section{Implications for Human Studies}

Unlike the PCI measured in humans, which is calibrated based on subjective reports (Casali et al. 2013; Casarotto et al. 2016), sPCI has obviously no relevance for consciousness; nonetheless, it provides a fundamental link between macroscale-level measurements and microscale neuronal events. At the macroscale level, the complex pattern of causal interactions triggered by TMS in awake humans collapses during sleep into a low complexity response associated with a stereotypical slow wave (Massimini et al. 2005). Such low complexity state reflects in part the local functional connectivity dominating the network during slow wave sleep or deep anesthesia (Bettinardi et al. 2015), which may be relevant for the functions of slow wave sleep (for a review see Diekelmann and Born 2010). At the mesoscale level, simultaneous intracortical ES and LFP recordings in humans suggest that the slow wave evoked during sleep might be associated with a Down state followed by a break-off of deterministic interactions (Pigorini et al. 2015). Crucially, the present microscale level exploration directly links neuronal bistability to perturbational complexity and further demonstrates that by manipulating the first it is possible to affect the latter. 
These findings are relevant for understanding how corticocortical information integration changes from wakefulness to NREM sleep, but may also have important implications for brain-injured patients. Indeed, cortical slices could be considered a simplified model of the electrophysiological state of cortical circuits under conditions of severe deafferentation, such as the ones associated with major brain lesions. Following traumatic brain insults, subcortical lesions, and diffuse axonal injuries may leave large portions of the cerebral cortex largely devoid of ascending inputs, including noradrenergic and cholinergic projections (Giacino et al. 2014). Beyond a critical level, such disconnection may force cortical circuits-that are otherwise intact-into a bistable, low-complexity state. In this way, some types of brain lesions may impair cortical information integration, above and beyond the associated anatomical disconnection. Clearly, understanding and promoting the mechanisms of such recovery is very important. In the simplified slice model tested here, the ability to sustain causal interactions and complex patterns of activity could be restored by a manipulation that selectively reduced neuronal bistability. In this perspective, the microscale model of perturbational complexity in the slice may represent a platform for testing increasingly selective pharmacological neuromodulation for defining novel therapeutic approaches aimed at brain-injured patients in whom brain complexity and consciousness are impaired in spite of preserved cortical activity.

\section{Supplementary Material}

Supplementary material is available at Cerebral Cortex online.

\section{Funding}

EU FP7-ICT CORTICONIC (Contract no. 600806) and the European Union's Horizon 2020 Research and Innovation Programme under Grant Agreement No. 720270 (HBP SGA1) (M. M. and M.V.S-.V.), Ministerio de Economía y Competividad (Spain) (BFU2014-52467-R to M.V.S-.V.) and CERCA Programme/ Generalitat de Catalunya. B.R. was supported by FPI fellowship BES-2012-053122 (MINECO, Spain).

\section{Notes}

We thank Simone Sarasso of University of Milan for his suggestions on the interpretation of results and on the manuscript writing, Lorena Pérez-Méndez for data analysis and Pedro Mateos-Aparicio and Almudena Barbero for their data contribution during the last stages of the study. Conflict of Interest: None declared.

\section{References}

Aghajanian GK, Rasmussen K. 1989. Intracellular studies in the facial nucleus illustrating a simple new method for obtaining viable motoneurons in adult rat brain slices. Synapse. 3(4):331-338.

Amigo JM, Monetti R, Tort-Colet N, Sanchez-Vives MV. 2015. Infragranular layers lead information flow during slow oscillations according to information directionality indicators. J Comput Neurosci. 39:53-62.

Bassett DS, Bullmore ET. 2009. Human brain networks in health and disease. Curr Opin Neurol. 22:340-347.

Bettinardi RG, Tort-Colet N, Ruiz-Mejias M, Sanchez-Vives MV, Deco G. 2015. Gradual emergence of spontaneous correlated brain activity during fading of general anesthesia in rats: evidences from fMRI and local field potentials. Neuroimage. 114:185-198.

Bullmore E, Sporns O. 2009. Complex brain networks: graph theoretical analysis of structural and functional systems. Nat Rev Neurosci. 10:186-198.

Casali AG, Gosseries O, Rosanova M, Boly M, Sarasso S, Casali KR, Casarotto S, Bruno MA, Laureys S, Tononi G, et al. 2013. A theoretically based index of consciousness independent of sensory processing and behavior. Sci Transl Med. 5: 198 ra105.

Casarotto S, Comanducci A, Rosanova M, Sarasso S, Fecchio M, Napolitani M, Pigorini A, Casali G, Trimarchi A, Boly PD, et al. 2016. Stratification of unresponsive patients by an independently validated index of brain complexity. Ann Neurol. 80:718-729.

Chauvette S, Crochet S, Volgushev M, Timofeev I. 2011. Properties of slow oscillation during slow-wave sleep and anesthesia in cats. J Neurosci. 31:14998-15008.

Chauvette S, Volgushev M, Timofeev I. 2009. Origin of active states in local neocortical networks during slow sleep oscillation. Cereb Cortex. 20:2660-2674.

Chon K, Scully CG, Lu S. 2009. Approximate entropy for all signals. IEEE Eng Med Biol Mag. 28:18-23.

Compte A, Reig R, Descalzo VF, Harvey MA, Puccini GD, Sanchez-Vives MV. 2008. Spontaneous high-frequency (10$80 \mathrm{~Hz}$ ) oscillations during up states in the cerebral cortex in vitro. J Neurosci. 28:13828-13844.

Compte A, Sanchez-Vives MV, McCormick DA, Wang XJ. 2003. Cellular and network mechanisms of slow oscillatory activity $(<1 \mathrm{~Hz})$ and wave propagations in a cortical network model. J Neurophysiol. 89:2707-2725.

Constantinople CM, Bruno RM. 2011. Effects and mechanisms of wakefulness on local cortical networks. Neuron. 69: 1061-1068.

Contreras D, Timofeev I, Steriade M. 1996. Mechanisms of longlasting hyperpolarizations underlying slow sleep oscillations in cat corticothalamic networks. J Physiol. 494(Pt 1): 251-264.

Cunningham MO, Davies $\mathrm{CH}$, Buhl EH, Kopell N, Whittington MA. 2003. Gamma oscillations induced by kainate receptor activation in the entorhinal cortex in vitro. J Neurosci. 23: 9761-9769.

Cunningham MO, Pervouchine DD, Racca C, Kopell NJ, Davies CH, Jones RS, Traub RD, Whittington MA. 2006. Neuronal metabolism governs cortical network response state. Proc Natl Acad Sci USA. 103:5597-5601.

Deco G, Tononi G, Boly M, Kringelbach ML. 2015. Rethinking segregation and integration: contributions of whole-brain modelling. Nat Rev Neurosci. 16:430-439.

Diekelmann S, Born J. 2010. The memory function of sleep. Nat Rev Neurosci. 11(2):114-126.

Ferrarelli F, Massimini M, Sarasso S, Casali A, Riedner BA, Angelini G, Tononi G, Pearce RA. 2010. Breakdown in cortical effective connectivity during midazolam-induced loss of consciousness. Proc Natl Acad Sci USA. 107:2681-2686.

Foehring RC, Schwindt PC, Crill WE. 1989. Norepinephrine selectively reduces slow $\mathrm{Ca} 2+-$ and $\mathrm{Na}+$-mediated $\mathrm{K}+$ currents in cat neocortical neurons. J Neurophysiol. 61: 245-256.

Holcman D, Tsodyks M. 2006. The emergence of up and down states in cortical networks. PLoS Comput Biol. 2:e23.

Giacino JT, Fins JJ, Laureys S, Schiff ND. 2014. Disorders of consciousness after acquired brain injury: the state of the science. Nat Rev Neurol. 10:99-114. 
Illa X, Rebollo B, Gabriel G, Sanchez-Vives MV, Villa R. 2015. A SU-8 based flexible microprobe for close and distal recordings from the cortical network. SPIE mictrotechologies. 951803-1803.

Jones BE. 2004. Activity, modulation and role of basal forebrain cholinergic neurons innervating the cerebral cortex. Prog Brain Res. 145:157-169.

Kaspar F, Schuster HG. 1987. Easily calculable measure for the complexity of spatiotemporal patterns. Phys Rev A. 36: 842-848.

Koch C, Massimini M, Boly M, Tononi G. 2016. Neural correlates of consciousness: progress and problems. Nat Rev Neurosci. 17:307-321.

Luczak A, Bartho P, Marguet SL, Buzsaki G, Harris KD. 2007. Sequential structure of neocortical spontaneous activity in vivo. Proc Natl Acad Sci USA. 104:347-352.

Lv J, Simpson DM, Bell SL. 2007. Objective detection of evoked potentials using a bootstrap technique. Med Eng Phys. 29: 191-198.

Mann EO, Kohl MM, Paulsen O. 2009. Distinct roles of GABA(A) and $G A B A(B)$ receptors in balancing and terminating persistent cortical activity. J Neurosci. 29:7513-7518.

Massimini M, Ferrarelli F, Huber R, Esser SK, Singh H, Tononi G. 2005. Breakdown of cortical effective connectivity during sleep. Science. 309:2228-2232.

Massimini M, Ferrarelli F, Esser SK, Riedner BA, Huber R, Murphy M, Peterson MJ, Tononi G. 2007. ). Triggering sleep slow waves by transcranial magnetic stimulation. Proc Natl Acad Sci USA. 104:8496-8501.

Massimini M, Ferrarelli F, Sarasso S, Tononi G. 2012. Cortical mechanisms of loss of consciousness: insight from TMS/EEG studies. Arch Ital Biol. 150:44-55.

Mattia M, Del Giudice P. 2002. Population dynamics of interacting spiking neurons. Phys Rev E Stat Nonlin Soft Matter Phys. 66:051917.

Mattia M, Sanchez-Vives MV. 2012. Exploring the spectrum of dynamical regimes and timescales in spontaneous cortical activity. Cogn Neurodyn. 6:239-250.

Pigorini A, Sarasso S, Proserpio P, Szymanski C, Arnulfo G, Casarotto S, Fecchio M, Rosanova M, Mariotti M, Lo Russo G, et al. 2015. Bistability breaks-off deterministic responses to intracortical stimulation during non-REM sleep. Neuroimage. 112:105-113.

Pincus SM, Goldberger AL. 1994. Physiological time-series analysis: what does regularity quantify? Am J Physiol. 266: H1643-H1656.

Ray S, Crone NE, Niebur E, Franaszczuk PJ, Hsiao SS. 2008. Neural correlates of high-gamma oscillations $(60-200 \mathrm{~Hz})$ in macaque local field potentials and their potential implications in electrocorticography. J Neurosci. 28:11526-11536.

Reig R, Mattia M, Compte A, Belmonte C, Sanchez-Vives MV. 2010. Temperature modulation of slow and fast cortical rhythms. J Neurophysiol. 103:1253-1261.
Rosanova M, Gosseries O, Casarotto S, Boly M, Casali AG, Bruno MA, Mariotti M, Boveroux P, Tononi G, Laureys S, et al. 2012. Recovery of cortical effective connectivity and recovery of consciousness in vegetative patients. Brain. 135:1308-1320.

Sakata S, Harris KD. 2009. Laminar structure of spontaneous and sensory-evoked population activity in auditory cortex. Neuron. 64:404-418.

Sanchez-Vives MV. 2012. Spontaneous Rhythmic Activity in the Adult Cerebral Cortex In Vitro. In: Ballanyi K, editor. Isolated Central Nervous System Circuits. Vol. 73. 1st ed. New York (NY): Humana Press. p. 263-284. Neuromethods Series.

Sanchez-Vives MV, Mattia M. 2014. Slow wave activity as the default mode of the cerebral cortex. Arch Ital Biol. 152: 147-155.

Sanchez-Vives MV, Massimini M, Mattia M. 2017. Shaping the default activity pattern of the cortical network. Neuron. doi: NEURON-D-16-02060R2.

Sanchez-Vives MV, Mattia M, Compte A, Perez-Zabalza M, Winograd M, Descalzo VF, Reig R. 2010. Inhibitory modulation of cortical up states. J Neurophysiol. 104:1314-1324.

Sanchez-Vives MV, McCormick DA. 2000. Cellular and network mechanisms of rhythmic recurrent activity in neocortex. Nat Neurosci. 3:1027-1034.

Schmidt SL, Chew EY, Bennett DV, Hammad MA, Frohlich F. 2013. Differential effects of cholinergic and noradrenergic neuromodulation on spontaneous cortical network dynamics. Neuropharmacology. 72:259-273.

Schwindt PC, Spain WJ, Crill WE. 1989. Long-lasting reduction of excitability by a sodium-dependent potassium current in cat neocortical neurons. J Neurophysiol. 61:233-244.

Seth AK, Barrett AB, Barnett L. 2011. Causal density and integrated information as measures of conscious level. Philos Trans A Math Phys Eng Sci. 369:3748-3767.

Shu Y, Hasenstaub A, McCormick DA. 2003. Turning on and off recurrent balanced cortical activity. Nature. 423:288-293.

Sinkkonen J, Tiitinen H, Naatanen R. 1995. Gabor filters: an informative way for analysing event-related brain activity. J Neurosci Methods. 56:99-104.

Sporns O, Tononi G, Edelman GM. 2000. Connectivity and complexity: the relationship between neuroanatomy and brain dynamics. Neural Netw. 13:909-922.

Steriade M, Nunez A, Amzica F. 1993. A novel slow ( $<1 \mathrm{~Hz})$ oscillation of neocortical neurons in vivo: depolarizing and hyperpolarizing components. J Neurosci. 13:3252-3265.

Timofeev I, Grenier F, Bazhenov M, Sejnowski TJ, Steriade M. 2000. Origin of slow cortical oscillations in deafferented cortical slabs. Cereb Cortex. 10:1185-1199.

Tononi G, Edelman GM. 1998. Consciousness and complexity. Science. 282:1846-1851.

Tononi G, Sporns O, Edelman GM. 1994. A measure for brain complexity: relating functional segregation and integration in the nervous system. Proc Natl Acad Sci USA. 91: 5033-5037. 